9q and 20, where, respectively, chromosomal loss and gain is frequently detected in SCA III ovarian tumors $(7,8)$. After selecting the genes on these respective chromosomes, hierarchical clustering is performed. Sorting the genes on chromosomal order reveals particular chromosomal regions with under- or overexpressed genes in subsets of SCA III tumors. Possible retention or amplification on chromosome $9 p$ in all tumors and predominant chromosomal loss on chromosome $9 \mathrm{q}$ in a number of SCA III tumors is observed. When analyzed in the same way, chromosome 20 reveals potential amplified regions in the majority of tumors (Figure 1, B and C).

Application of this method gives direct insight into the chromosomal distribution of up- or down-regulated genes. It reveals continuous regions with gene expression biases. The visualization of these biases is a first step in better understanding of the relations between physical loss or gain of chromosomal regions and the effects of gene expression of genes located in these regions. This observation may also affect the significance of microarray expression data. Up-regulating of a gene within a region of other upregulated genes may be due to genomic instability and can be less significant than a solitary up-regulated gene. The macro and the user manual are available from the authors at http://cms-dev. bioasp.nl/applicationservices/extra/ genesort?view=MoreDetail and also as supplementary material at the BioTechniques web site at http://www.BioTechniques.com/April04/vanEijkSupplementary.html.

\section{ACKNOWLEDGMENTS}

This work was supported by the Leiden University Medical Center. We thank Dr. Han Rauwerda and the Bioinformatics Application Service Provider (BioASP) for making the guide available through the BioASP.

\section{REFERENCES}

1.Kallioniemi, A., O.P. Kallioniemi, D. Sudar, D. Rutovitz, J.W. Gray, F. Waldman, and D. Pinkel. 1992. Comparative genomic hybridization for molecular cytogenetic analysis of solid tumors. Science 258:818-821.

2.Crawley, J.J. and K.A. Furge. 2002. Identification of frequent cytogenetic aberrations in hepatocellular carcinoma using gene-expression microarray data. Genome Biol. 3:RESEARCH0075.

3.Caron, H., B. van Schaik, M. van der Mee, F. Baas, G. Riggins, P. van Sluis, M.C. Hermus, R. van Asperen, et al. 2001. The human transcriptome map: clustering of highly expressed genes in chromosomal domains. Science 29:1289-1292.

4.Pollack, J.R., T. Sorlie, C.M. Perou, C.A. Rees, S.S. Jeffrey, P.E. Lonning, R. Tibshirani, D. Botstein, et al. 2002. Microarray analysis reveals a major direct role of DNA copy number alteration in the transcriptional program of human breast tumors. Proc. Natl. Acad. Sci. USA 99:12963-12968.

5.Hughes, T.R., C.J. Roberts, H. Dai, A.R. Jones, M.R. Meyer, D. Slade, J. Burchard, S. Dow, et al. 2000. Widespread aneuploidy revealed by DNA microarray expression profiling. Nat. Genet. 25:333-337.

6.Harding, M.A., K.C. Arden, J.W. Gildea, J.J. Gildea, E.J. Perlman, C. Viars, and D. Theodorescu. 2002. Functional genomic comparison of lineage-related human bladder cancer cell lines with differing tumorigenic and metastatic potentials by spectral karyotyping, comparative genomic hybridization, and a novel method of positional expression profiling. Cancer Res. 62:6981-6989.

7.Hu, J., V. Khanna, M.W. Jones, and U. Surti. 2003. Comparative study of primary and recurrent ovarian serous carcinomas: comparative genomic hybridization analysis with a potential application for prognosis. Gynecol. Oncol. 89:369-375.

8.Bayani, J., J.D. Brenton, P.F. Macgregor, B. Beheshti, M. Albert, D. Nallainathan, J. Karaskova, B. Rosen, et al. 2002. Parallel analysis of sporadic primary ovarian carcinomas by spectral karyotyping, comparative genomic hybridization, and expression microarrays. Cancer Res. 62:3466-3476.

9.Irizarry, R.A., B. Hobbs, F. Collin, Y.D. Beazer-Barclay, K.J. Antonellis, U. Scherf, and T.P. Speed. 2003. Exploration, normalization, and summaries of high density oligonucleotide array probe level data. Biostatistics 4:249-264.

Received 14 November 2003; accepted 11 February 2004.

Address correspondence to Ronald van Eijk, Department of Pathology, Leiden University Hospital, Albinusdreef 2, Building 1, P1-41, P.O. Box 9600, 2300 RC Leiden, The Netherlands.e-mail: R.van_Eijk@lumc.nl

\title{
Production and assay of a transcription activator CRP in coupled in vitro transcription and translation
}

\author{
Shumo Liu ${ }^{1}$ and Albert Libchaber ${ }^{2}$ \\ ${ }^{1}$ NEC Laboratories America, Princeton, NJ and ${ }^{2}$ Rockefeller University, New York, \\ NY, USA
}

BioTechniques 36:596-600 (April 2004)

We describe a method to produce an Escherichia coli transcription activator and assay its function in vitro in a coupled transcription and eukaryotic translation (CTT) reaction. The model transcription activator used here is CRP (also called CAP), a well-known $E$. coli catabolic regulator protein. In the presence of cyclic-AMP (cAMP), the CRP-cAMP complex binds to a CRPdependent promoter, interacts with RNA polymerase and stimulates transcription at the promoter (1-4).
In the experiments described here, we use T3 RNA polymerase to produce CRP in vitro from a plasmid, pT3CRP, which has the CRP gene, crp, transcribed from a T3 promoter (Figure 1). The function of CRP is tested with a reporter plasmid, p42crp-luc, which has a synthetic CRP-dependent promoter and the firefly luciferase gene, luc. The synthetic promoter has the following features: (i) it has a -10 site of $E$. coli consensus promoter to ensure a high promoter activity upon activation; (ii) 


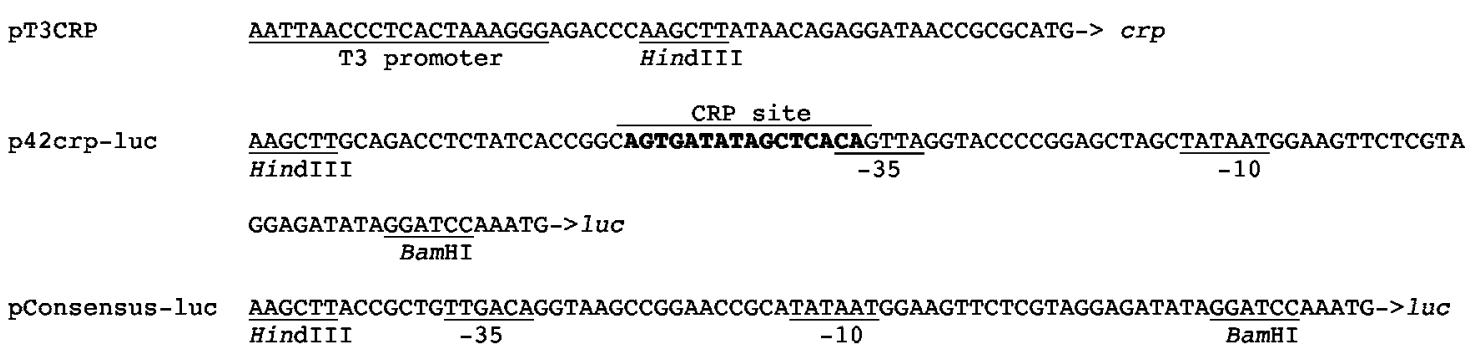

Figure 1. Promoter regions of the plasmids used in experiments. Each sequence includes the promoter region to the first codon of the gene. Only one strand is shown. Promoter sites are underlined. The catabolic regulator protein (CRP) binding site is in bold. Also underlined are some restriction sites. The ribosome binding sites are not particularly designed. The $c r p$ is obtained from an Escherichia coli K-12 strain by PCR. The plasmids are constructed on a pGEM-type plasmid vector.
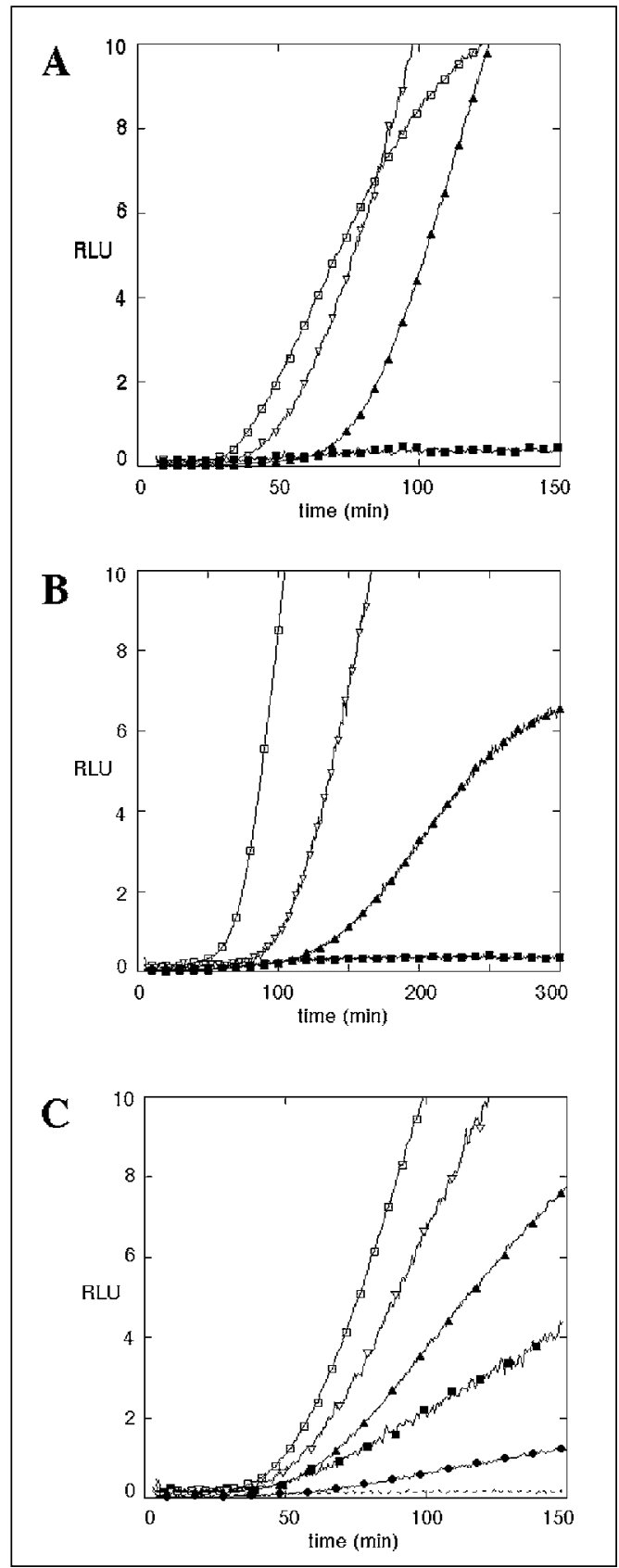

598 BioTechniques it lacks a functional -35 site so that the basal activity is low; and (iii) it has a CRP-binding site centered at -41.5 position. This ensemble constitutes a class II CRP-dependent $E$. coli promoter $(5,6)$. The expression of $l u c$ from the promoter can be monitored in a CTT reaction with a luminometer (7).

The CTT reaction uses a coupled transcription and translation kit ${\left(\mathrm{TNT}^{\circledR}\right.}^{\circledR} \mathrm{T} 3$ Coupled Wheat Germ Extract System; Promega, Madison, WI, USA), which uses wheat germ extract for translation. The components in the kit, excluding RNA polymerase, are first mixed according to the instructions. Luciferin (Promega) is added to the mixture to $0.4 \mathrm{mM}$ (from a 20
$\mathrm{mM}$ stock solution in $10 \mathrm{mM}$ Tris- $\mathrm{Cl}$, $\mathrm{pH} 8.0$, stored at $-70^{\circ} \mathrm{C}$ ). For sampleto-sample uniformity, this CTT mixture is prepared batch-wise, divided in aliquots, and stored at $-70^{\circ} \mathrm{C}$. The transcription activity assay reaction is made of $10 \mu \mathrm{L}$ CTT mixture plus $0.5 \mathrm{U}$ $\sigma^{70}$-saturated $E$. coli RNA polymerase (Epicentre, Madison, WI, USA), 0.03 $\mathrm{nM}$ reporter plasmid DNA, and other ingredients (specified below). The final concentration is about $10 \%$ lower than that recommended for CTT by the kit. At this slightly lowered concentration, the reaction is less sensitive to volume variation. To improve precision, reactions of four activation conditions are carried out in parallel. The common ingredients, such as reporter plasmid DNA and RNA polymerase, are first mixed in a larger volume. The larger volume is then di-

Figure 2. Expression of $l u c$ under various transcription activation conditions. The transcription activity is reported by luc expression, which in turn, is measured by the light output from the reaction with a luminometer in relative luminescence units (RLUs). Four photomultiplier tubes (PMTs) (type H5784; Hamamatsu, Hamamatsu, Japan) are used to simultaneously monitor four reactions. The output of a PMT (pulses of approximately $5 \mathrm{mV}$ and $5 \mu$ s wide) is fed to a pulse integration amplifier. The integration time is about $10 \mathrm{~s}$. The amplifier's output (V) is recorded at 30-s intervals with a computer-based data acquisition system from National Instruments (Austin, TX, USA) and plotted later. The voltage is proportional to the light intensity and is used as RLU. The sensitivities of the channels are calibrated to within 5\% from each other at full range. (A) Activation by catabolic regulator protein (CRP) at CRP-dependent promoter. Each reaction contains Escherichia coli RNA polymerase. Open square, $0.03 \mathrm{nM}$ pConsensusluc; open triangle, $0.03 \mathrm{nM}$ p $42 \mathrm{crp}-1 \mathrm{cuc}, 3 \%$ premade CRP, and $2 \mu \mathrm{M}$ cAMP. Filled triangle, 0.03 nM p42crp-luc, $0.075 \mathrm{nM}$ pT3CRP, $2 \%$ (v/v) T3 RNA polymerase, and $2 \mu \mathrm{M}$ cAMP. Filled square, same as filled triangle but without cAMP. (B) Activation at CRP-dependent promoter as a function of pT3CRP concentration. Each reaction has E. coli RNA polymerase, T3 RNA polymerase, p42crp-luc, and $2 \mu \mathrm{M}$ cAMP. The concentrations of pT3CRP DNA are: open square, $0.075 \mathrm{nM}$; open triangle, $0.0075 \mathrm{nM}$; filled triangle, $0.00075 \mathrm{nM}$; and filled square, $0 \mathrm{nM}$. The decreasing concentration of pT3CRP DNA is made by serial dilution of the reactions. (C) Activation by CRP as a function of cAMP concentration. Each reaction contains $E$. coli RNA polymerase, p42crp-luc, and 3\% premade CRP. cAMP is added as follows: open square, $2 \mu \mathrm{M}$; open triangle, $0.8 \mu \mathrm{M}$; filled triangle, $0.6 \mu \mathrm{M}$; filled square, $0.2 \mu \mathrm{M}$; filled circle, $0.13 \mu \mathrm{M}$; and dash line, $0 \mu \mathrm{M}$. The reactions shown are from 12 reactions in three measurement groups. Reactions of $2,0.2$, and $0 \mu \mathrm{M}$ are from one group; 0.6 and $0.13 \mu \mathrm{M}$ are from another; $0.8 \mu \mathrm{M}$ is from still another. For clarity, six other reactions are not shown. 
vided to the four individual reactions to which other ingredients specific to each reaction are added.

At the time of experiment, the reactions are prepared in clear $0.2-\mathrm{mL}$ PCR tubes at $0^{\circ} \mathrm{C}$. The temperature of the tubes is raised to room temperature $\left(22^{\circ}-23^{\circ} \mathrm{C}\right)$ to start CTT. Although the CTT reaction temperature can be raised to at least $30^{\circ} \mathrm{C}$, we choose room temperature for convenience. Each reaction is monitored with a photomultiplier tube (PMT) of a four-channel luminometer. The relative luminescence unit (RLU) is recorded with a computer. The transcription level of luc can be estimated from RLU values. The variation of RLU is around $10 \%$ among repeated reactions.

Functional CRP can be produced and assayed either in two separate CTT reactions (referred to as premade (RP) or in a combined reaction (referred to as in situ activation). Premade CRP is produced in $10 \mu \mathrm{L}$ CTT reaction with 2\% (v/v) T3 RNA polymerase (Promega; included in the CTT kit) and $0.15 \mathrm{nM}$ pT3CRP DNA. The reaction lasts for $100 \mathrm{~min}$ at room temperature, and it is terminated by adding $5 \mu \mathrm{L}$ glycerol and stored at $-20^{\circ} \mathrm{C}$ as premade CRP preparation. In the following experiment, we use $3 \%(\mathrm{v} / \mathrm{v})$ premade CRP preparation and $2 \mu \mathrm{M}$ cAMP (Sigma, St. Louis, MO, USA) in an assay reaction (Figure $2 \mathrm{~A}$ ). For in situ activation, we add T3RNA polymerase $(2 \%, \mathrm{v} / \mathrm{v})$ and pT3CRP DNA $(0.075 \mathrm{nM})$ directly to another assay reaction. As a reference, we include a positive control reaction with pConsensus-luc. This plasmid has a constitutively active consensus promoter in place of the CRP-dependent promoter on p42crp-luc (Figure 1). Also included is a negative control reaction with premade CRP but without cAMP.

Figure 2A shows promoter activity of reactions as reported by $l u c$ expression. In the presence of cAMP and CRP, the CRP-dependent promoter activity is as active as the consensus promoter. CRP can be supplied to the promoter either in situ or premade. In the case of in situ activation, there is an additional lag of luc expression, which reflects the time needed to produce enough CRP. In contrast to the active state, in the ab- sence of cAMP (negative control) CRPdependent promoter gives very low luc expression. Therefore, the transcription at the CRP-dependent promoter is clearly cAMP dependent, confirming the identity of CRP as the activator protein. The low level of $l u c$ expression in negative control also shows that wheat germ extract has no detectable amount of either endogenous cAMP or cAMPindependent activator.

Next, we tested in situ activation dependence on pT3CRP DNA concentration (Figure 2B). The luc expression is approximately proportional to the concentration of pT3CRP. Evidently CRPdependent promoter activity is proportional to CRP concentration, which in turn, is proportional to pT3CRP concentration. The very low luc expression without p3TCRP indicates that wheat germ extract has no detectable amount of endogenous CRP-like activator. Finally, we observe CRP activation at various concentrations of cAMP (Figure $2 \mathrm{C}$ ). The activity at $2 \mu \mathrm{M}$ cAMP is near maximum (higher than $2 \mu \mathrm{M}$ cAMP not shown), and the half-activation concentration of cAMP is between 0.6 and $0.8 \mu \mathrm{M}$. The cAMP dose response is comparable to what has been previously reported $(8,9)$.

In the experiments, we apply CTT to produce CRP from its DNA coding sequences and to observe CRP transcription activation in near real-time. We use wheat germ extract to avoid the interference from endogenous CRP and cAMP, which are usually present in $E$. coli 30-S extract. Combining activator production and assay in one, the reaction mimics a simple in vivo gene expression circuit.

\section{REFERENCES}

1.Kolb, A., S. Busby, H. Buc, S. Garges, and S. Adhya. 1993. Transcriptional regulation by cAMP and its receptor protein. Annu. Rev. Biochem. 62:749-795.

2.Busby, S. and R.H. Ebright. 1999. Transcription activation by catabolite activator protein (CAP). J. Mol. Biol. 293:199-213.

3.Harman, J.G. 2001. Allosteric regulation of the cAMP receptor protein. Biochim. Biophys. Acta 1547:1-17.

4.Savery, N., V. Rhodius, and S. Busby. 1996. Protein-protein interactions during transcription activation: the case of the Escherichia coli cyclic AMP receptor protein. Philos. Trans. R Soc. Lond. B Biol. Sci. 351:543-550.
5.Ebright, R.H. 1993. Transcription activation at Class I CAP-dependent promoters. Mol. Microbiol. 8:797-802.

6.Ushida, C. and H. Aiba. 1990. Helical phase dependent action of CRP: effect of the distance between the CRP site and the -35 region on promoter activity. Nucleic Acids Res. 18:6325-6330

7.Kolb, V.A., E.V. Makeyev, and A.S. Spirin. 1994. Folding of firefly luciferase during translation in a cell-free system. EMBO J. 13:3631-3637.

8.Nisseley, S.P., W.B. Anderson, M.E. Gottesman, R.L. Perlman, and I. Pastan. 1971. In vitro transcription of the gal operon requires cyclic adenosine monophosphate and cyclic adenosine monophosphate receptor protein. $\mathrm{J}$. Biol. Chem. 246:4671-4678.

9.Takahashi, M., B. Blazy, A. Baudras, and W. Hillen. 1989. Ligand-modulated binding of a gene regulatory protein to DNA. Quantitative analysis of cyclic-AMP induced binding of CRP from Escherichia coli to non-specific and specific DNA targets. J. Mol. Biol. 207:783-976.

Received 2 September 2003; accepted 11 February 2004.

Address correspondence to Shumo Liu, Department of Physics, University of California at San Diego, 9500 Gilman Drive, MC0374, La Jolla, CA 92093, USA. e-mail: sliu@physics.ucsd.edu 